Math. Model. Nat. Phenom.

Vol. 3, No. 4, 2008, pp. 131-160

\title{
Possibly Longest Food Chain: Analysis of a Mathematical Model
}

\author{
T. Matsuoka and H. Seno ${ }^{1}$ \\ Department of Mathematical and Life Sciences, Graduate School of Science \\ Hiroshima University \\ Kagamiyama 1-3-1, Higashi-hiroshima, 739-8526 Japan
}

\begin{abstract}
We consider the number of trophic levels in a food chain given by the equilibrium state for a simple mathematical model with ordinary differential equations which govern the temporal variation of the energy reserve in each trophic level. When a new trophic level invades over the top of the chain, the chain could lengthen by one trophic level. We can derive the condition that such lengthening could occur, and prove that the possibly longest chain is globally stable. In some specific cases, we find that the possibly longest chain is such that the lower trophic level has a greater energy reserve than the higher has, so that the distribution of energy reserves can be regarded to have a pyramid shape, whereas, if any of its trophic levels is removed, the pyramid shape cannot be maintained. Further, we find the condition that arbitrary long chain can be established. In such unbounded case, we prove that any chain could not have the pyramid shape of energy reserve distribution.
\end{abstract}

Key words: food chain; energy reserve; trophic level; mathematical model.

AMS subject classification: 92D40, 92D25, 92B05, 70K99

\section{Introduction}

It is one of the important issues in ecology to identify the general properties about the structure of food web, as theoretically studied by many researchers (for instance, [3, 4, 10, 13, 19, 21, 28, $29,30,31,33,34])$. The length of food chain is one of the important features interesting for such theoretical studies $[23,25,31]$. There are some different ways to measure the length of food chain

\footnotetext{
${ }^{1}$ Corresponding author. E-mail: seno@math.sci.hiroshima-u.ac.jp
}

\section{1}


and some hypotheses about what determines the length of food chain [17, 19, 23, 24, 25, 31]. One method to estimate the length of food chain is to deal with the energy flow, which is sometimes called the realized trophic position [23]. It represents how many times the energy (or a certain material) is transferred from a primary producer to a consumer. Then the average number of links from each producer to each top predator is regarded as defining the length of food chain. In this case, we need to calculate/estimate the number of trophic links about all trophic pathways that lead from primary producers to top predators.

The network of energy flows in a food web could be theoretically simplified to a linear chain of energy flows. Such a theoretical framework was mathematically discussed by Higashi et al. [10]. Along their theory, we could resolve and reconstruct the network of energy flows in a food web into some linear chains. Teramoto [32] analyzed the following model of an energy food chain with $m$ energy trophic levels:

$$
\begin{aligned}
\frac{d N_{m, m}}{d t}=\nu \alpha N_{m-1, m} N_{m, m}-\left(\delta+\theta N_{m, m}\right) N_{m, m} ; \\
\frac{d N_{i, m}}{d t}=\nu \alpha N_{i-1, m} N_{i, m}-\left(\delta+\theta N_{i, m}\right) N_{i, m}-\alpha N_{i, m} N_{i+1, m} \\
\quad(3 \leq i \leq m-1) ; \\
\frac{d N_{2, m}}{d t}=\mu \beta N_{1, m} N_{2, m}-\left(\delta+\theta N_{2, m}\right) N_{2, m}-\alpha N_{2, m} N_{3, m} ; \\
\frac{d N_{1, m}}{d t}=\varepsilon\left(1-N_{1, m}\right) N_{1, m}-\beta N_{1, m} N_{2, m},
\end{aligned}
$$

where $N_{i, m}=N_{i, m}(t)(i=1,2, \ldots, m)$ is the energy reserve of the $i$ th trophic level at time $t$. Parameters $\alpha, \beta, \delta, \theta, \mu$ and $\nu$ are all positive constants. $\mu$ and $\nu$ are the successful energy fixation rate for the second trophic level (herbivore) and that for the higher trophic levels (carnivores) respectively. $\alpha$ is the energy transfer rate from the $i-1$ th trophic level to the $i$ th $(i=3,4, \ldots, m)$. The primary energy production is given by the logistic growth term with the intrinsic growth rate $\varepsilon$ and the unity of carrying capacity. $\delta$ is the intrinsic energy dissipation rate at each trophic level. $\theta$ introduces the intra-trophic density effect to increase the energy dissipation at each trophic level. Harrison [9] considered the global stability of the equilibrium state for the more general system including (1.1). He proved that the equilibrium state with the positive energy reserve at every trophic level is globally stable. In contrast, some theoretical researches indicate that the long food chain of a wide family of mathematical models would have a chaotic parameter region [6].

Teramoto [32] considered a specific case if the model (1.1) when $\mu \beta=\nu \alpha$ for the second to the third trophic levels, and obtained the following results:

- A finite upper bound for the number of trophic levels exists.

- The number of trophic levels of the longest chain has a positive correlation with $\nu \alpha / \delta$ and $\varepsilon$.

- In the longest chain, the distribution of energy reserves among trophic levels is always such that the lower trophic level has a greater energy reserve than the higher has, in other words, it has a pyramid shape. 
- For sufficiently large $\theta$, the pyramid shape can be maintained even if the top trophic level is removed from the equilibrium state with a pyramid shape of the energy reserve distribution.

A similar and different food chain model was discussed from the similar viewpoint in Chapter 5 of Svirezhev and Logofet [31]. Differently from (1.1), no density effect in the energy input or the energy dissipation are included, that is, the primary trophic level is given by

$$
\frac{d N_{1, m}}{d t}=\phi-\beta N_{1, m} N_{2, m}
$$

where the primary energy production rate is given by a constant $\phi$, and $\theta=0$.

In this paper, we consider a mathematical model of an energy food chain simpler than (1.1), and similar to that in [31]. Our model does not incorporate any density effect within each trophic level. We focus the number of trophic levels in the possibly longest chain and try to discuss the lengthening of the chain by the invasion of a trophic level over the present top level.

\section{Model}

We consider the following system which is similar to and different from those in Chapter 5 of Svirezhev and Logofet [31] and Gurney and Nisbet [7]. It governs the temporal variation of energy reserves in the food chain with $m$ energy trophic levels, which we call hereafter the $m$ level system (Fig. 1):

$$
\begin{aligned}
& \frac{d N_{m, m}}{d t}= \alpha_{m} N_{m-1, m} N_{m, m}-\delta_{m} N_{m, m} ; \\
& \frac{d N_{i, m}}{d t}= \alpha_{i} N_{i-1, m} N_{i, m}-\delta_{i} N_{i, m}-\alpha_{i+1} N_{i, m} N_{i+1, m} \\
& \quad(2 \leq i \leq m-1) ; \\
& \frac{d N_{1, m}}{d t}=\phi-\delta_{1} N_{1, m}-\alpha_{2} N_{1, m} N_{2, m},
\end{aligned}
$$

where $N_{i, m}=N_{i, m}(t)$ is the energy reserve of the $i$ th trophic level at time $t$. Parameters $\alpha_{i}, \delta_{i}$, and $\phi$ are all positive constants. $\alpha_{i}$ is the energy transfer rate from the $i-1$ th trophic level to the $i$ th, $\delta_{i}$ the energy dissipation rate at the $i$ th trophic level. The primary energy production rate is given by a constant $\phi$. We do not consider the density effect within each trophic level.

In case of the food chain, the energy transfer is realized by the predation, that is, by the intertrophic reaction with the interspecific reaction. In this sense, the model should have a reaction term between subsequent trophic levels. In our model, it is given by the mass-action term which is a simplest form to introduce such an reaction. Besides the interaction term must be zero if one of subsequent trophic levels is zero, because the interaction never occurs between them. The mass-action term is the simplest form satisfying this nature.

Some knowledges about the natures of the simplest system could serve to understand some characteristics of the more complicated system, and furthermore would sometimes give some perspectives for the ecological problem to be discussed. 


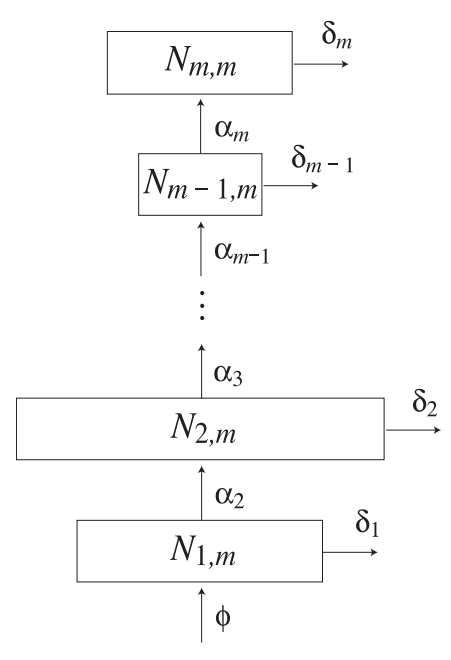

Figure 1: Illustration of the $m$ level system given by (2.1).

\section{Existence of equilibrium states}

For the $m$ level system, we define the $k$ level established state $(k<m)$ as the equilibrium state at which those levels from the first to the $k$ th are positive and the others zero:

$$
\left(N_{1, m}^{*}, N_{2, m}^{*}, \ldots, N_{m, m}^{*}\right)=(\underbrace{+,+, \ldots,+}_{k}, \underbrace{0, \ldots, 0}_{m-k})
$$

where $N_{i, m}^{*}$ is the equilibrium value of the $i$ th level in the $m$ level system. The completely established state is specifically defined as the equilibrium state at which every trophic level has a positive equilibrium value for the $m$ level system.

From (2.1), we can explicitly obtain every equilibrium value of the $k$ level established state (Appendix A): For even $k$,

$$
\begin{array}{rll}
N_{2 i, m}^{*} & =\frac{1}{\alpha_{2 i} R_{i-1} Q_{k}}\left(\phi-P_{2 i} Q_{k}\right) & (2 \leq i \leq k / 2) \\
N_{2 i+1, m}^{*} & =\frac{\delta_{2(i+1)}}{\alpha_{2(i+1)}}+\sum_{l=i+1}^{k / 2-1} \frac{\delta_{2(l+1)}}{\alpha_{2(l+1)}} \frac{R_{i}}{R_{l}} & (1 \leq i \leq k / 2-2) ; \\
N_{2, m}^{*} & =\frac{\phi / \alpha_{2}}{Q_{k}}-\frac{\delta_{1}}{\alpha_{2}} \\
N_{1, m}^{*} & =Q_{k},
\end{array}
$$




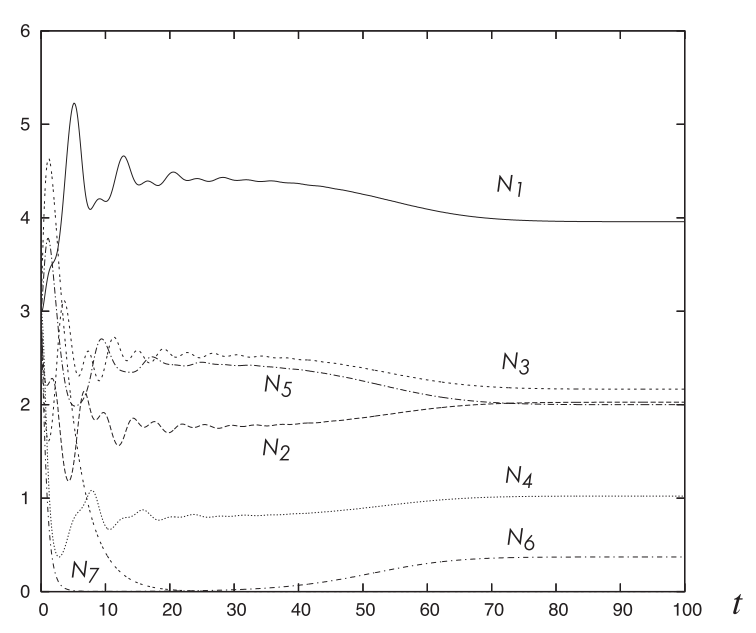

Figure 2: A numerical calculation for the seven level system with $\alpha_{2}=0.4 ; \alpha_{3}=0.5 ; \alpha_{4}=0.6$; $\alpha_{5}=0.5 ; \alpha_{6}=0.3 ; \alpha_{7}=0.4 ; \delta_{1}=0.2 ; \delta_{2}=0.5 ; \delta_{3}=0.4 ; \delta_{4}=0.3 ; \delta_{5}=0.4 ; \delta_{6}=0.6 ;$ $\delta_{7}=0.3 ; \phi=4.0 ; N_{i, 7}(0)=3.0(i=1,2, \ldots, 7) . P_{6} Q_{6}=3.58<\phi<P_{7} Q_{7}=4.43$. The six level established state is globally stable, the seventh trophic level going extinct.

and for odd $k$,

$$
\begin{array}{rlr}
N_{2 i+1, m}^{*} & =\frac{R_{i}}{P_{k}}\left(\phi-P_{k} Q_{2 i}\right) & (2 \leq i \leq(k-1) / 2) ; \\
N_{2 i, m}^{*} & =\frac{\delta_{2 i+1}}{\alpha_{2 i+1}}+\sum_{l=i+1}^{(k-1) / 2} \frac{\delta_{2 l+1} R_{l}}{\alpha_{2 i+1} R_{i}} & (1 \leq i \leq(k-3) / 2) ; \\
N_{3, m}^{*} & =\frac{\alpha_{2}}{\alpha_{3}} \frac{\phi}{P_{k}}-\frac{\delta_{2}}{\alpha_{3}} ; \\
N_{1, m}^{*} & =\frac{\phi}{P_{k}},
\end{array}
$$

where

$$
\begin{aligned}
& R_{l}=\prod_{j=1}^{l} \frac{\alpha_{2 j}}{\alpha_{2 j+1}} ; \\
& P_{k}=\delta_{1}+\sum_{l=1}^{\llbracket(k-1) / 2 \rrbracket} \delta_{2 l+1} R_{l} \quad(k \geq 3) ; P_{1}=P_{2}=\delta_{1} ; \\
& Q_{k}=\frac{\delta_{2}}{\alpha_{2}}+\sum_{l=1}^{\llbracket k / 2-1 \rrbracket} \frac{\delta_{2(l+1)}}{\alpha_{2(l+1)}} \frac{1}{R_{l}} \quad(k \geq 4) ; \quad Q_{2}=Q_{3}=\frac{\delta_{2}}{\alpha_{2}} ; Q_{1}=0 .
\end{aligned}
$$

Bracket $\llbracket \cdot \rrbracket$ denotes the Gauss's symbol such that $\llbracket a \rrbracket$ gives the maximal integer not beyond $a$.

Now, with regard to the existence of the $k$ level established state, we can obtain the following theorem (Appendix B): 
Theorem 1. For the $m$ level system, the $k$ level established state uniquely exists if and only if $\phi>P_{k} Q_{k}$. If $\phi<P_{k} Q_{k}$, it does not exist.

We note that the single level established state always exists with $N_{1, m}^{*}=\phi / \delta_{1}$. The two level established state exists if and only if $\phi>\delta_{1} \delta_{2} / \alpha_{2}$. At any equilibrium state, the trophic levels lower than a certain level are positive and the others zero (Lemma 12 in Appendix B). Theorem 1 indicates that the existence of the $k$ level established state requires the primary production rate $\phi$ greater than the critical value $P_{k} Q_{k}$ (see a numerical example in Fig. 2). Since $P_{k} Q_{k}$ is monotonically increasing in terms of $k$, the greater primary production rate could afford the longer chain. Similar results and discussions are given also for the similar model in Chapter 5 of Svirezhev and Logofet [31].

From Theorem 1, we can obtain the following corollaries (Appendix C):

Corollary 2. If the $k$ level established state exists, then the $j$ level established state exists for any $j<k$.

Corollary 3. The $k$ level established state exists if and only if the $k-1$ level established state exists with $N_{k-1, m}^{*}>\delta_{k} / \alpha_{k}$.

Corollary 2 means that the existence of a chain assures the existence of any chain shorter than it. Corollary 3 indicates that the existence of a longer chain requires a sufficiently large energy reserve at the top trophic level in the shorter chain.

\section{Stability of equilibrium states}

We can obtain the following theorem and corollaries about the stability of $k$ level established state (Appendix D):

Theorem 4. If the $k$ level established state exists and the $k+1$ level established does not, then the $k$ level established state is globally stable.

Corollary 5. If the completely established state exists, it is globally stable.

Corollary 6. If the $k$ level established state exists unstable, the $k+1$ level established state exists.

Theorem 4 and Corollary 5 mean that the possibly longest chain for a given $m$ level system is globally stable. From Corollary 6, if the $m-1$ level established state exists unstable, the completely established state exists globally stable. These corollaries give some suggestions about the lengthening of the energy food chain, which we discuss in the other section. 


\section{Length of the longest chain}

From Theorem 1, only if $P_{k} Q_{k}$ converges to a finite value as $k \rightarrow \infty$, arbitrary long chain for a given finite value of $\phi$ could be established. A long chain could be regarded to have grown by a large sequence of introductions of additional level over the top of chain. From definitions of $P_{k}$ and $Q_{k}$, both of $\left\{P_{k}\right\}$ and $\left\{Q_{k}\right\}$ are rigorously increasing sequences of positive numbers in terms of $k$. Thus, the convergence of $P_{k} Q_{k}$ is equivalent to that of both $P_{k}$ and $Q_{k}$ as $k \rightarrow \infty$. Therefore, we can obtain the following theorem:

Theorem 7. Only if both $P_{k}$ and $Q_{k}$ converge to finite values, $P$ and $Q$ respectively, as $k \rightarrow \infty$, then arbitrary long chain could be established with $\phi>P Q$. If $\phi<P Q$, the chain length has a finite upper bound.

Let us consider a specific case with $\alpha_{i}=\alpha_{2} \zeta^{i-2}$ and $\delta_{i}=\delta_{1} \xi^{i-1}(2 \leq i \leq m)$, where $\alpha_{2}, \delta_{1}$, $\zeta$, and $\xi$ are positive constants. From the definitions of $P_{k}$ and $Q_{k}$, in this case, $P_{k} Q_{k}$ converges to a finite value as $k \rightarrow \infty$ if and only if

$$
q=\frac{\xi^{2}}{\zeta}<1
$$

and we have

$$
P_{k} Q_{k} \rightarrow P Q=\frac{\delta_{1} \delta_{2}}{\alpha_{2}} \frac{1}{(1-q)^{2}} \quad \text { as } k \rightarrow \infty .
$$

Hence, when $q<1$ and $\phi>P Q$, arbitrary long chain can be established.

In case of $q<1$, the condition $\phi>P Q$ corresponds to

$$
\zeta>\frac{\xi^{2} \sqrt{\phi \alpha_{2}}}{\sqrt{\phi \alpha_{2}}-\delta_{1} \sqrt{\xi}}
$$

Inequality (5.3) gives the region UNBOUNDED in Fig. 3(a). From (5.3), we find that such specific case occurs only if $\xi<\phi \alpha_{2} / \delta_{1}^{2}$. If $\xi>\phi \alpha_{2} \delta_{1}^{2}$, it never occurs. If $\xi<\phi \alpha_{2} / \delta_{1}^{2}$, there is some $\zeta$ such that such specific case occurs. Therefore, the parameter $\xi$ is essential to determine if such specific case occurs or not.

Even when $q<1$, there is a finite upper bound for the number of trophic levels if $\phi<P Q$, which corresponds to the region FINITE1 in Fig. 3(a) (see also Fig. 4). In this case, we can explicitly derive the following finite number $m_{\max }$ of the trophic levels in the possibly longest chain (Appendix F):

$$
m_{\max }=\left\{\begin{array}{cl}
\llbracket \Lambda \rrbracket & \text { if } \llbracket \Theta \rrbracket \text { is even; } \\
\llbracket \Theta \rrbracket & \text { if } \llbracket \Theta \rrbracket \text { is odd, }
\end{array}\right.
$$

where

$$
\begin{aligned}
\Theta & =\frac{\ln \{1-(1-q) \rho / 2\}}{\ln \sqrt{q}} ; \\
\Lambda & =\frac{\ln \left\{1+q-(1-q) \sqrt{1+q \rho^{2}}\right\}-\ln 2}{\ln \sqrt{q}}-1,
\end{aligned}
$$


(a)

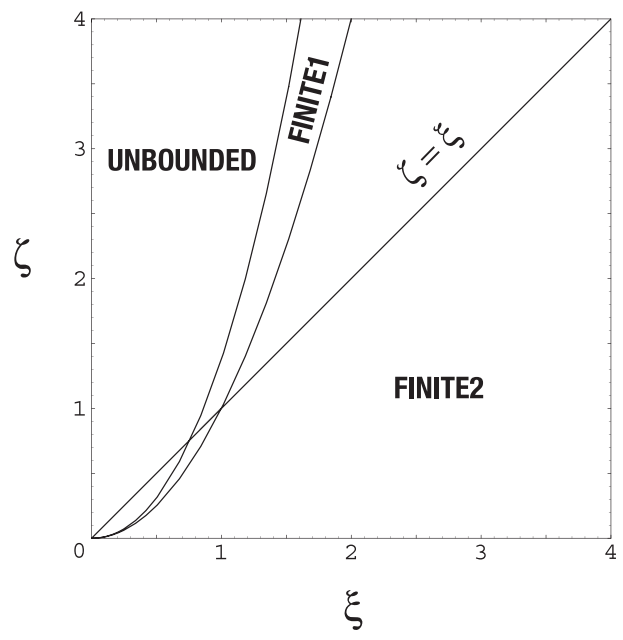

(b)

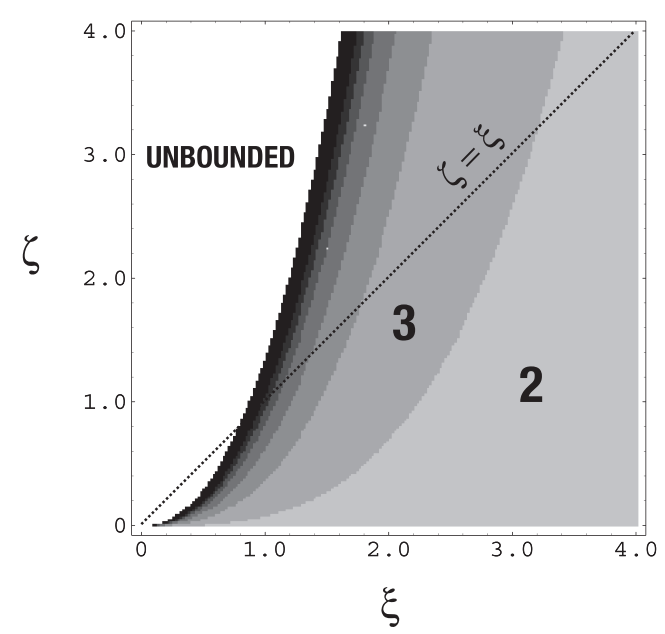

Figure 3: $(\xi, \zeta)$-dependence of the length of food chain when $\alpha_{i}$ and $\delta_{i}$ have geometric variations: $\alpha_{i}=\alpha_{2} \zeta^{i-2}$ and $\delta_{i}=\delta_{1} \xi^{i-1}$. (a) In the region UNBOUNDED, $P_{k} Q_{k}$ converges to a finite value $P Q$ as $k \rightarrow \infty$, satisfying that $\phi>P Q$. In the region FINITE1, the chain length has a finite upper bound although $P_{k} Q_{k}$ converges to a finite value $P Q$ as $k \rightarrow \infty$, satisfying $\phi<P Q$. In the region FINITE2, $P_{k} Q_{k}$ diverges as $k \rightarrow \infty$. (b) The finite upper bound $m_{\max }$ for the number of trophic levels: For the darker region, the upper bound $m_{\max }$ is larger. Numerically drawn with $\phi=2.4$, $\alpha_{2}=0.5$ and $\delta_{1}=0.3$. For $\xi \geq \phi \alpha_{2} / \delta_{1}^{2}=13.3$, only the single level established state exists. 

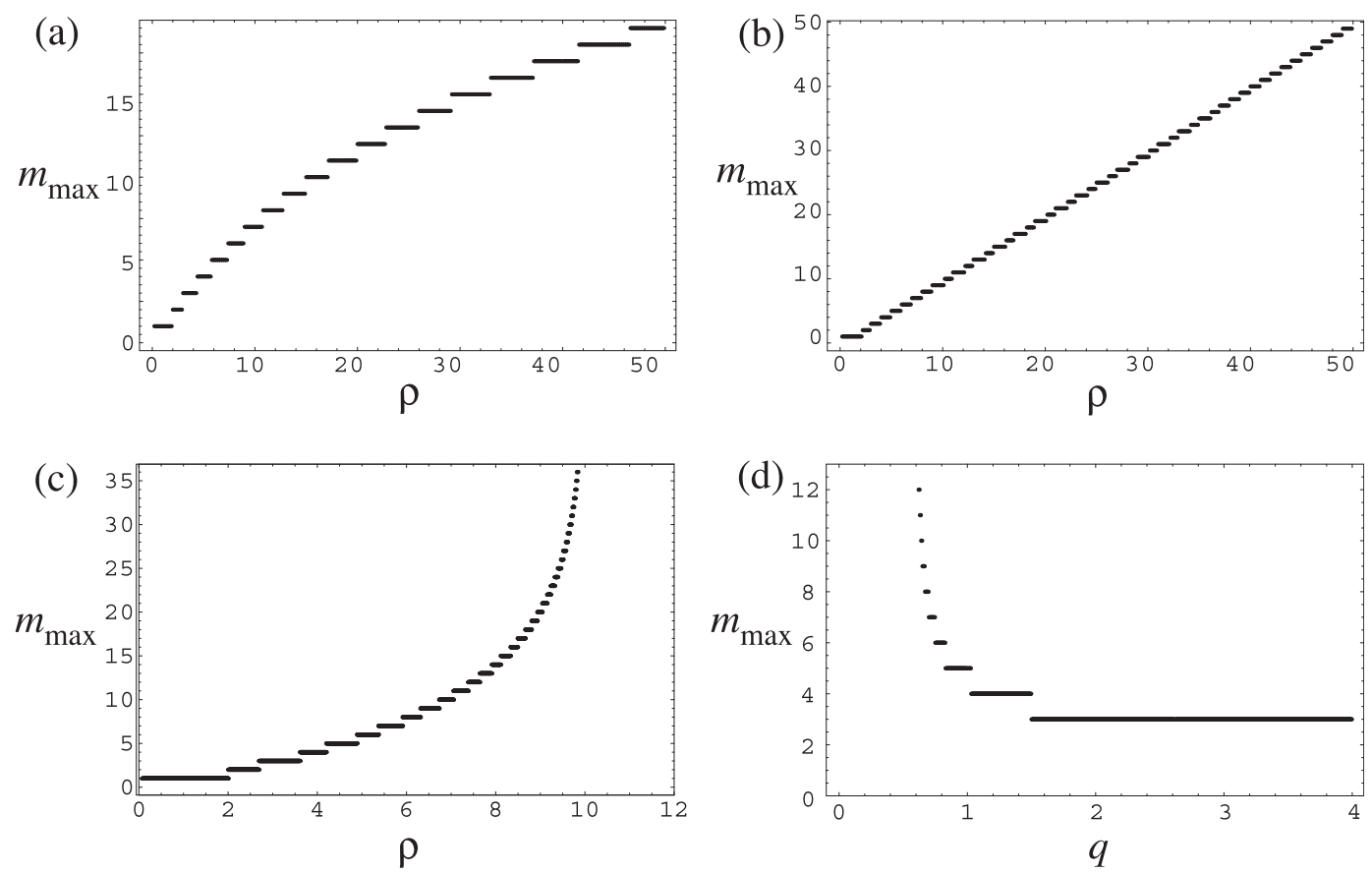

Figure 4: Parameter dependence of the number $m_{\max }$ of trophic levels in the possibly longest chain in case of $\alpha_{i}=\alpha_{2} \zeta^{i-2}$ and $\delta_{i}=\delta_{1} \xi^{i-1}$, making use of (5.4-5.7). $\rho=\sqrt{4 \alpha_{2} \phi /\left(\delta_{1} \delta_{2}\right)} ; q=\xi^{2} / \zeta$. (a-c) $\rho$-dependence; (d) $q$-dependence. (a) $q=1.2$; (b) $q=1$; (c) $q=0.8$; (d) $\rho=5.0$. In (c), $P_{k} Q_{k}$ converges to $P Q$ as $k \rightarrow \infty$ and $\phi>P Q$ when $\rho>2 /(1-q)=10.0$. In (d), $\phi>P Q$ when $q<1-2 / \rho=0.6$. 
with $\rho=\sqrt{4 \alpha_{2} \phi /\left(\delta_{1} \delta_{2}\right)}$. Bracket $\llbracket \cdot \rrbracket$ is the Gauss's symbol as before. It is easily shown that $\Lambda$ is positive whenever $\phi<P Q$.

For any $q>1$, as indicated by the region FINITE2 of $(\xi, \zeta)$-space in Fig. 3(a), we can find again the finite upper bound $m_{\max }$ given by (5.4). In case of $q=1$, we can find the following finite upper bound $m_{\max }$ (Appendix F):

$$
m_{\max }=\left\{\begin{array}{cl}
{\left[\sqrt{\rho^{2}+1}\right]} & \text { if } \llbracket \rho \rrbracket \text { is even; } \\
\llbracket \rho \rrbracket & \text { if } \llbracket \rho \rrbracket \text { is odd, }
\end{array}\right.
$$

where $\rho$ is the same as before. Consequently, if $q \geq 1$, the chain length necessarily has a finite upper bound (Fig. 4). The following condition is that the two level established state is the longest chain (see Fig. 3(b)):

$$
\frac{\delta_{1} \delta_{2}}{\alpha_{2}}<\phi<\frac{\delta_{1} \delta_{2}}{\alpha_{2}}(1+q) .
$$

The similar discussion was carried out for the similar model in Chapter 5 of Svirezhev and Logofet [31], too. They analyzed the case of $\xi=\zeta=1$ and showed that the arbitrary long chain cannot be established. The result is approved also for our model because $q=1$ in case of $\xi=\zeta=1$ in our model. In our model, we remark that, even when $q<1$, if $\phi<P Q$, the chain length has a finite upper bound (see Figs. 3 and 4).

\section{Invasion of an additional top trophic level}

In this section, we discuss the possibility to add a trophic level over the top of existing energy food chain. Such introduction of a new trophic level could be regarded as the invasion of an alien top predator into a food web. At first, we obtain the following lemma (Appendix E):

Lemma 8. If the $m+1$ th trophic level with $\delta_{m+1} / \alpha_{m+1}<N_{m, m}^{*}$ is introduced into the completely established state of the $m$ level system, then the completely established state becomes unstable.

Now suppose that the completely established state exists for the $m$ level system. From Corollary 6 and Lemma 8, if the $m+1$ th level with $\delta_{m+1} / \alpha_{m+1}<N_{m, m}^{*}$ is introduced, then the system changes to the $m+1$ level system, and the completely established state of the $m+1$ level system subsequently becomes globally stable (a numerical example is given in Fig. 5(a)). Lastly, we can get the following theorem:

Theorem 9. If the $m+1$ th trophic level with $\delta_{m+1} / \alpha_{m+1}<N_{m, m}^{*}$ is introduced into the completely established state of the $m$ level system, then the completely established state of the $m+1$ level system appears globally stable.

Therefore, the invasion of an alien top predator with sufficiently small ratio $\delta / \alpha$ can lengthen the food chain. It is implied that the establishment of a long food chain would require a sequence of invasions of alien top predator which has a sufficiently small ratio $\delta / \alpha$, that is, has a high efficiency of the energy fixation. 
(a)

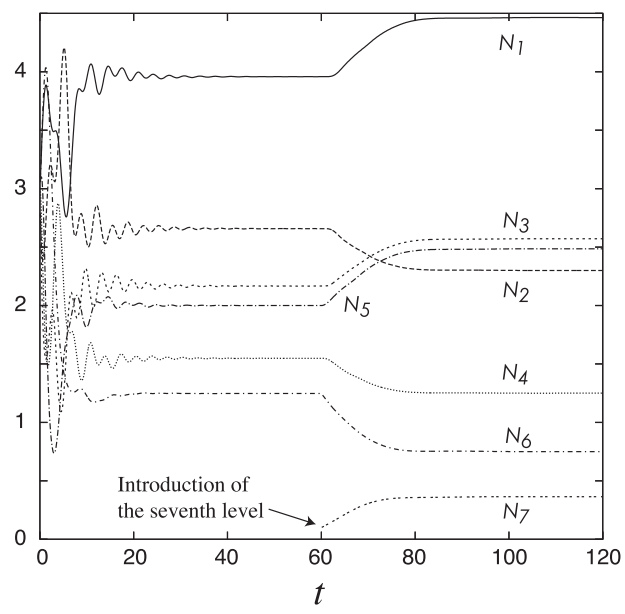

(b)

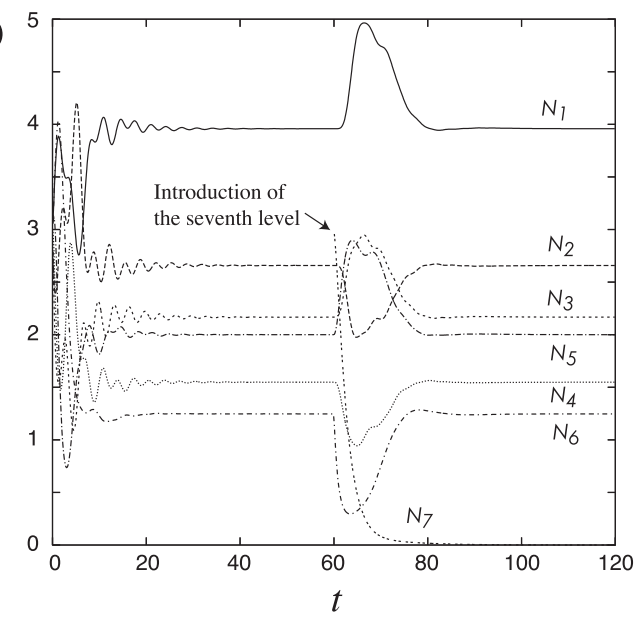

Figure 5: A numerical calculation about the effect of the seventh level introduction into the six level system. The seventh level is introduced at $t=60$. (a) $\alpha_{7}=0.4 ; \delta_{7}=0.3 ; \delta_{7} / \alpha_{7}=0.75<N_{6,6}^{*}=$ $1.25 ; N_{7,7}(60)=0.1$. (b) $\alpha_{7}=0.3 ; \delta_{7}=0.5 ; \delta_{7} / \alpha_{7}=1.67>N_{6,6}^{*}=1.25 ; N_{7,7}(60)=3.0$. In (a), the completely established state for the seven level system appears after the seventh level introduction. In (b), the introduction of the seventh level fails, and the completely established state for the six level system recovers. $\alpha_{2}=0.4 ; \alpha_{3}=0.5 ; \alpha_{4}=0.6 ; \alpha_{5}=0.5 ; \alpha_{6}=0.3 ; \delta_{1}=0.2$; $\delta_{2}=0.5 ; \delta_{3}=0.4 ; \delta_{4}=0.3 ; \delta_{5}=0.4 ; \delta_{6}=0.6 ; \phi=5.0 ; N_{i, 6}(0)=3.0(i=1,2, \ldots, 6)$.

\section{Distribution of energy reserves}

In this section, we consider the distribution of energy reserves at the equilibrium state of the $m$ level system. We obtain the following theorem (Appendix G):

Theorem 10. In the possibly longest chain which has $m_{\max }$ trophic levels for sufficiently narrow range of $\alpha_{i}$ and $\delta_{i}$, it is satisfied that $N_{i-1, m_{\max }}^{*}>N_{i, m_{\max }}^{*}$ for any $i \leq m_{\max }$.

Theorem 10 indicates that, for the possibly longest chain when parameters $\alpha$ and $\delta$ are different little among trophic levels, the distribution of energy reserves has a pyramid shape.

From Theorem 10, we can obtain the following corollary about the distribution of energy reserves at the equilibrium state which appears after the removal of a trophic level at the completely established state of the $m_{\max }$ level system with a pyramid shape of the energy reserve distribution (Appendix H):

Corollary 11. In the possibly longest chain which has $m_{\max }$ trophic levels for a sufficiently narrow range of $\alpha_{i}$ and $\delta_{i}$, if the $k$ th level $\left(3 \leq k \leq m_{\max }\right)$ is removed, the system transits to the $k-1$ level established state with an energy reserve distribution which satisfies the following characteristics: 
(a)

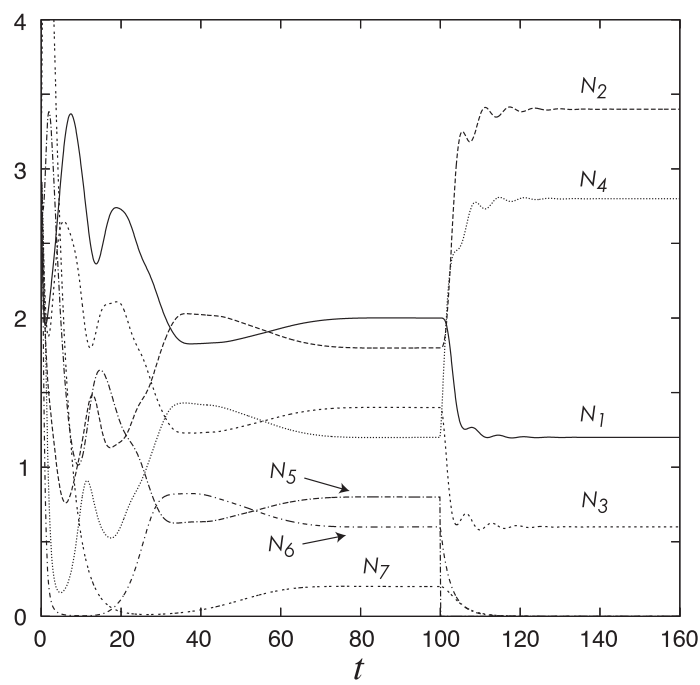

(b)

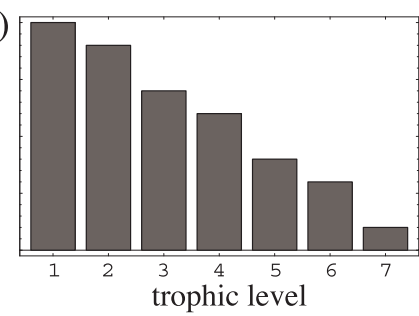

(c)

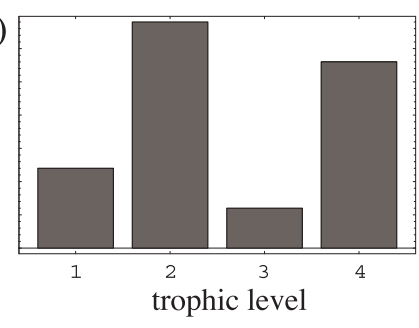

Figure 6: A numerical calculation of the seven level system. At $t=100, N_{5}$ is forced to change to zero, then all levels lower than the fifth go to the new equilibrium states. (a) the temporal variation of energy reserves; (b) the distribution of energy reserves at $t=95$; (c) that at $t=150 . \phi=2.4$; $\alpha_{i}=0.5 ; \delta_{i}=0.3 ; N_{i, 7}(0)=3.0(i=1,2, \ldots, 7) . P_{7} Q_{7}=2.16<\phi<2.88=P_{8} Q_{8}$.

For even $k$,

$$
\begin{array}{lll}
N_{2(i-1), k-1}^{*}< & N_{2 i-1, k-1}^{*} & (2 \leq i \leq k / 2) \\
N_{2(i-1), k-1}^{*} & >N_{2 i, k-1}^{*} & (2 \leq i \leq k / 2-1) \\
N_{2 i-1, k-1}^{*} & >N_{2 i+1, k-1}^{*} & (1 \leq i \leq k / 2-1)
\end{array}
$$

and for odd $k$,

$$
\begin{array}{clll}
N_{2 i-1, k-1}^{*} & < & N_{2 i, k-1}^{*} & (1 \leq i \leq(k-1) / 2) \\
N_{2(i-1), k-1}^{*} & > & N_{2 i, k-1}^{*} & (2 \leq i \leq(k-1) / 2) \\
N_{2 i-1, k-1}^{*} & > & N_{2 i+1, k-1}^{*} & (1 \leq i \leq(k-3) / 2) .
\end{array}
$$

This corollary indicates the destruction of a pyramid shape of the energy reserve distribution in the possibly longest chain by the removal of a trophic level. In the same time, it is indicated that any established state shorter than $m_{\max }$ cannot have a pyramid shape of the energy reserve distribution in this case. We give a numerical example in Fig. 6.

Let us consider again the specific case of $\alpha_{i}=\alpha_{2} \zeta^{i-2}$ and $\delta_{i}=\delta_{1} \xi^{i-1}(i \geq 2)$. We numerically investigate the $(\xi, \zeta)$-dependence of the energy reserve distribution in the possibly longest chain, and get the result as shown in Fig. 7. For convenience, we used the following monotonicity index $I_{\triangle}$ which reflects the degree of the monotonicity of the energy reserve distribution for the possibly 

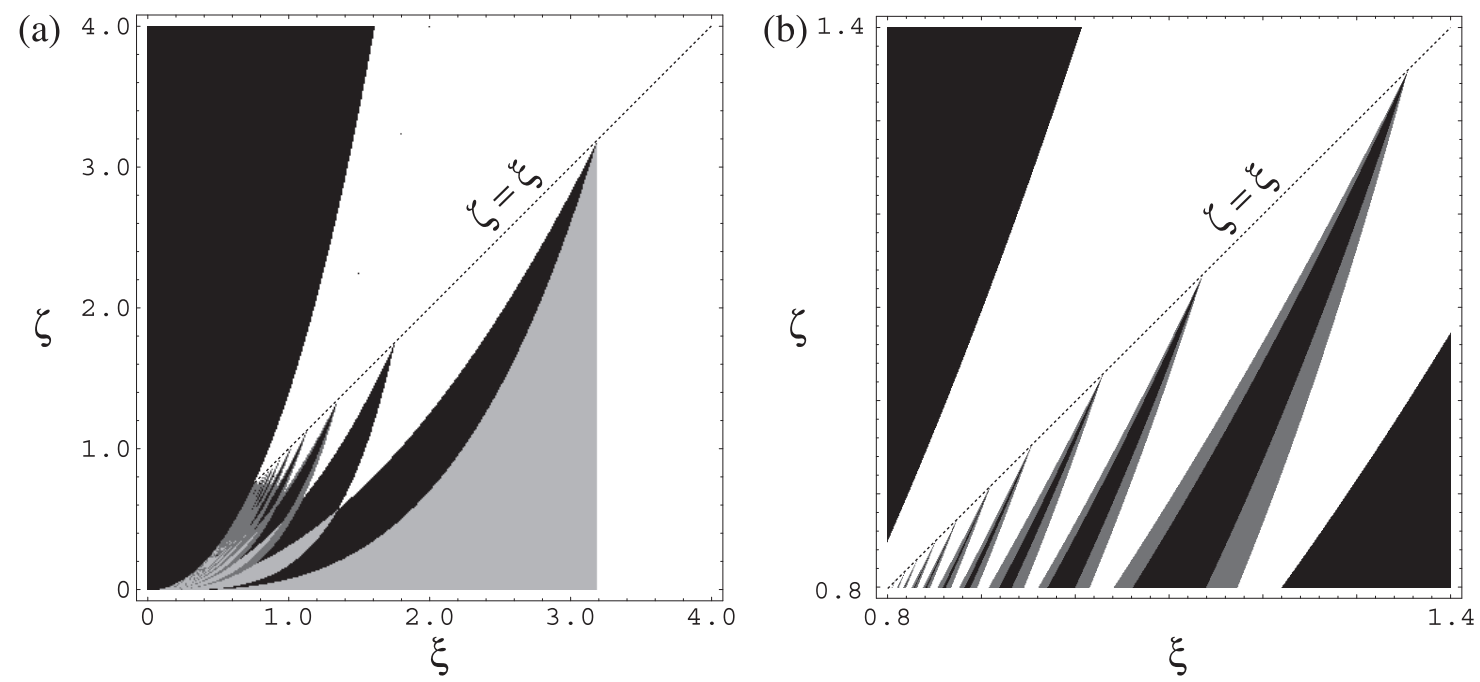

Figure 7: $(\xi, \zeta)$-dependence of the monotonicity of the energy reserve distribution. Numerically obtained for the case of $\alpha_{i}=\alpha_{2} \zeta^{i-2}$ and $\delta_{i}=\delta_{1} \xi^{i-1}(i \geq 2)$. (a) $(\xi, \zeta) \in[0,4.0] \times[0,4.0]$; (b) $[0.8,1.4] \times[0.8,1.4]$. White region is for the monotonicity index $I_{\triangle}=1$ with a pyramid shape of the energy reserve distribution. Light dark region is for $I_{\triangle}=1$ with an inverted pyramid shape of the energy reserve distribution. The black region is for $I_{\triangle}=-1$, and the medium dark region for $\left|I_{\triangle}\right|<1 . \phi=2.4 ; \alpha_{2}=0.5 ; \delta_{1}=0.3$. For $\xi \geq \phi \alpha_{2} / \delta_{1}^{2}=13.3$, only the single level established state exists.

(a)

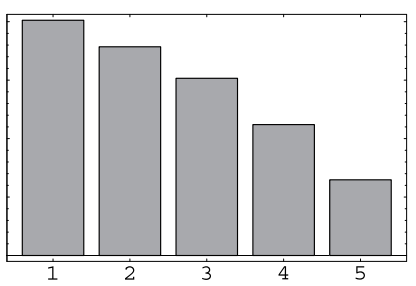

(d)

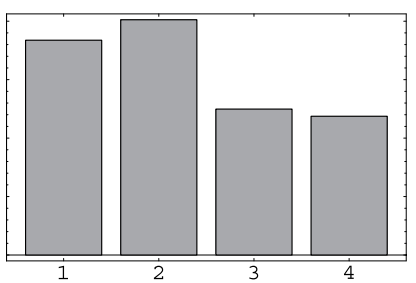

(b)

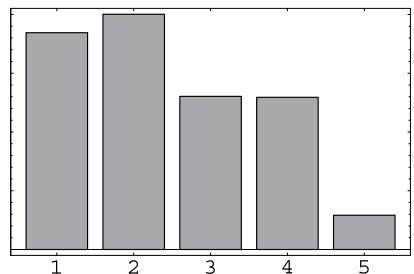

(e)

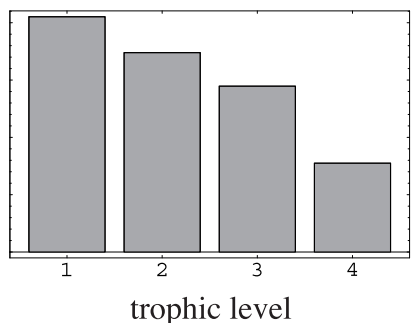

(c)

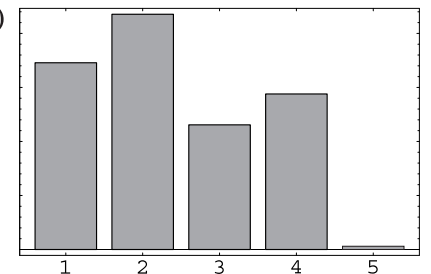

(f)

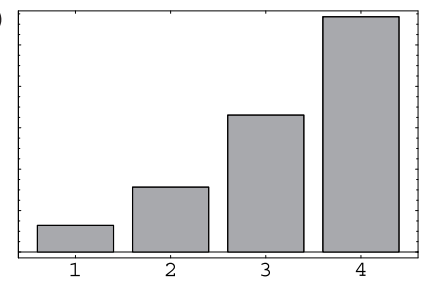

Figure 8: Energy reserve distribution of the possibly longest chain. Numerically obtained for the case of $\alpha_{i}=\alpha_{2} \zeta^{i-2}$ and $\delta_{i}=\delta_{1} \xi^{i-1}(i \geq 2)$. (a) $\xi=1.08$ and $I_{\triangle}=1$; (b) $\xi=1.12$ and $I_{\triangle}=1 / 3$; (c) $\xi=1.15$ and $I_{\triangle}=-1$; (d) $\xi=1.17$ and $I_{\triangle}=0$; (e) $\xi=1.25$ and $I_{\triangle}=1$; (f) $\xi=0.6$ and $I_{\triangle}=1$. (a-e) $\zeta=0.9$ (f) $\zeta=0.14$. Commonly, $\phi=2.4 ; \alpha_{2}=0.5 ; \delta_{1}=0.3$. 
longest chain with $m_{\max }$ trophic levels $\left(m_{\max }>2\right)$ :

$$
I_{\triangle}=\frac{1}{m_{\max }-2} \sum_{i=2}^{m_{\max }-1} \operatorname{sgn}\left[\left(N_{i+1, m_{\max }}^{*}-N_{i, m_{\max }}^{*}\right)\left(N_{i, m_{\max }}^{*}-N_{i-1, m_{\max }}^{*}\right),\right.
$$

where $\operatorname{sgn}[x]$ is 1 for $x \geq 0$ and -1 for $x<0$. If and only if the energy reserve distribution has a pyramid shape or an inverted pyramid shape, the monotonicity index $I_{\triangle}$ is 1 . If $I_{\triangle}$ is less than 1 , the distribution has some non-monotonical parts.

As indicated by Figs. 7 and 8, the appearance of a pyramid shape of the energy reserve distribution in the possibly longest chain has a non-simple relation to $(\xi, \zeta)$. It is indicated that the possibly longest chain would not necessarily has a pyramid shape of the energy reserve distribution, and the change of the chain length would easily disrupt the pyramid shape if it exists before the change. Exceptionally, in the case when the longest chain consists of only two trophic levels, the energy reserve of the first level is greater than that of the second if and only if the parameter $\xi$ is large enough to satisfy that $\phi<\xi(1+\xi) \delta_{1}^{2} / \alpha_{2}$ (see also Fig. 3(b)).

We can analytically show that a pyramid shape of the energy reserve distribution appears for the possibly longest chain if $\xi / \zeta \leq 1$ (Appendix I; also see Fig. 7). It could appear when the possibly longest (finite) chain has a ratio of the dissipation rates between subsequent levels smaller than that of the energy transfer rates. We numerically find that the pyramid shape could not appear when $q<1$ and $\phi>P Q$, even if $\xi / \zeta \leq 1$, as shown by the black region in Fig. 7. We can prove that, when arbitrary long chain could exist, the pyramid shape of the energy reserve distribution cannot appear for any sufficiently long chain with $m$ trophic levels $(m \gg 1)$ (Appendix J).

As for the scaling property appeared in Fig. 7, although it would be interesting from a mathematical viewpoint, we can just give a possible conjecture that the condition for the monotonicity of the energy reserve distribution may depend on the parameters $\xi$ and $\zeta$, for instance, as a power ratio $\xi^{a} / \zeta^{b}$ only which magnitude plays a role to determine the monotonicity, because they contribute as a scaling relation numerically shown in Fig. 7. We do not discuss the scaling property in depth any more since it must depend on the concrete $i$-dependence of $\alpha_{i}$ and $\beta_{i}$, and may not be essential for the framework of our discussion in this paper.

\section{Discussion}

Our results indicate that the greater primary production rate could establish the longer food chain. Some previous researches show that there would be some food chains in which the primary production rate would determine the length $[12,36]$. This is formalized and discussed as the productive space hypothesis $[23,25,26]$.

Although the possibly longest chain is globally stable, it could be lengthened by the invasion of an alien top predator which has a sufficiently small ratio of the energy dissipation rate and its transfer rate. Hence, in a sufficiently long chain, the higher trophic levels might be expected to have a small ratio $\delta / \alpha$ : In such a long chain, the higher trophic level would be composed of species which have a high efficiency of the energy fixation with a low dissipation rate and a high energy gain rate. 
If each of the energy transfer rate and the dissipation rate has a value similar among all trophic levels, then there is a finite upper bound for the number of trophic levels, and the food chain could not be lengthened beyond it by the invasion of an alien top predator which has similar dissipation and energy transfer rates. The analogous result is shown in Chapter 5 of Svirezhev and Logofet [31] and in Teramoto [32].

In this paper, we considered the specific case when each of the energy transfer rate and the dissipation rate has a geometric variation in the food chain. In this case, we explicitly demonstrate that the energy reserve efficiency of each trophic level determines the food chain length as discussed in $[5,31,35]$. However, some researches say that the resource availability does not directly determine the food chain length in most natural systems [11, 23, 25, 27]. As for our model, when the square of energy dissipation rate becomes sufficiently smaller than the energy transfer rate at the higher trophic levels, the arbitrary long chain could be established. In such case, we may suggest that other factors which are not involved in our model would determine the food chain length. Similar discussion about whether the resource availability essentially limits the food chain length or not have been on the table [18, 20, 23, 25].

Lindeman [15] considered the progressive efficiency $\lambda_{i} / \lambda_{i-1}$ about the food chain dynamics, where $\lambda_{i}$ is the energy in-flow rate into the $i$ th trophic level:

$$
\frac{d N_{i, m}}{d t}=\lambda_{i}-\lambda_{i}^{\prime}
$$

where $\lambda_{i}^{\prime}$ is the out-flow rate. Lindeman [15] suggests that the progressive efficiency gets larger for the higher trophic level because animals in the higher trophic level could more efficiently search their food. In our model, the progressive efficiency corresponds to $\alpha_{i} N_{i-1, m}^{*} / \alpha_{i-1} N_{i-2, m}^{*}$ at the equilibrium state. On the other hand, the pyramid shape of the energy reserve distribution is often observed in nature [16]. Some of recent researches say that the biomass abundance would be constant among species, while the number of species would be decreasing in terms of trophic levels (for instance, see [2]). From this viewpoint, the pyramid shape of energy reserve distribution would be generally observed. In case of the pyramid shape of energy reserve distribution, we have $N_{i-1, m}^{*} / N_{i-2, m}^{*}<1$. So, if the progressive efficiency is large for the higher trophic level, it is necessary that the ratio $\alpha_{i} / \alpha_{i-1}$ is sufficiently larger than 1 . In our case of geometrically variable $\alpha_{i}$, this is the case of $\zeta>1$. Moreover, in our result, a sufficiently small $\xi$ is necessary for the appearance of a pyramid shape of the energy reserve distribution. Therefore, we could suggest that a pyramid shape of the energy reserve distribution would appear when the energy transfer rate gets larger at the higher trophic level while the energy dissipation rate is not so large as the transfer rate.

Although the longest chain always has a pyramid shape of the energy reserve distribution for the model in Teramoto [32], it does not for our model. According to our model, a pyramid shape of the energy reserve distribution appears for the possibly longest chain if the dissipation and the energy transfer rates of every trophic level are similar values throughout the chain. However, in case of geometric variations of the dissipation and the energy transfer rates, it appears particularly when the dissipation rate is sufficiently smaller than the energy transfer rate at any trophic level. It does not appear even for the possibly longest chain in some cases. These results suggest that the pyramid shape of the energy reserve distribution would not be generally observable. In Teramoto 
[32], a pyramid shape could appear even for the shorter chain if the density effect within each trophic level is strong enough. Therefore, we could suggest that the density effect may play an essential role to cause a pyramid shape of the energy reserve distribution.

The food chain in nature has been exposed to the invasions of alien top predators and the exchanges of the member species. The length of food chain must have been temporally changing in a sufficiently large time scale. Nevertheless, if a pyramid shape of the energy reserve distribution could be observed in nature, those results of Teramoto [32] and ours suggest that the density effect would play an important role to regulate the distribution. Moreover, if some exchanges of member species in a trophic level occur, the parameter values characterizing each trophic level may change, and consequently the length of food chain and the nature of energy reserve distribution may change due to the instability of the chain structure.

Some other models predict that an alien top predator with sufficiently large growth rate could easily invade a food chain (for instance, see [14]). Then it is implied that a food chain with a moderate growth rate of the top predator would be easily invaded by an alien top predator which grows sufficiently fast. On the other hand, it is suggested that the length of the possibly longest chain would be at most 12 (with the mean around 7) [8, 22]. Hutchinson [11] mentioned that predators in the higher trophic level would get bigger. Larger organisms commonly grow more slowly so that their populations have the smaller growth rate. Thus, the invasion of an alien top predator into the existing food chain would be hardly successful in nature, so that the much long chain might be rare in nature. In our model, the long chain cannot be established with the great energy dissipation rate. If the predators in the higher trophic level would need to compensate the large energy dissipation rate by some strategy, it requires a cost for the persistence with the other species including their preys. Such a cost might be the reason why the food chain length in nature could not be so long.

In our model, we used the mass-action term to introduce the inter-trophic reaction. It is not only because of the mathematically simplest form to be considered as the first step of research but also because of its possible role to get the fundamental nature of the system under consideration, and moreover because of its potentiality to catch the essential features which could appear in the more sophisticated model with the other type of inter-trophic reaction term belonging to a family of functional forms. For instance, some other food chain model with the different type of intertrophic reaction may have a non-static stable state (e.g. a temporally periodic or chaotic solution), as indicated by some theoretical researches (for instance, [6]). However, as for the model (1.1) by Teramoto [32] with a linearly density-dependent rate of energy dissipation in each trophic level, we do not think that there would be any significant difference in the theoretical results, that is, we think that our model could bring the essentially same results with those in [32], as already mentioned in the above.

We did not consider the temporally varying environments (for example, seasonal variation) whereas it would be an important factor to determine the length of food chain [1]. The primary production rate would be particularly sensitive to the environmental variation. Since our result indicates that the primary production rate is one of the important factors to determine the food chain length, such an environmental variation would cause a temporal change of the length, accompanying the immigration or the emigration/extinction of some top predators. 


\section{Appendix}

\section{A Relations among equilibrium values}

From (2.1), we can obtain the following relations among equilibrium values of the $k$ level established state for the $m$ level system:

$$
\begin{aligned}
& N_{j, m}=0 \quad(j>k) ; \\
& N_{k, m}^{*}=\frac{\phi-\sum_{i=1}^{k-1} \delta_{i} N_{i, m}^{*}}{\delta_{k}} ; \\
& N_{k-1, m}^{*}=\frac{\delta_{k}}{\alpha_{k}} ; \\
& N_{2, m}^{*}=\frac{\delta_{3}+\alpha_{4} N_{4, m}^{*}}{\alpha_{3}}=\frac{\phi-\delta_{1} N_{1, m}^{*}}{\alpha_{2} N_{1, m}^{*}} ; \\
& N_{1, m}^{*}=\frac{\phi}{\delta_{1}+\alpha_{2} N_{2, m}^{*}}=\frac{\delta_{2}+\alpha_{3} N_{3, m}^{*}}{\alpha_{2}}
\end{aligned}
$$

and for even $k$,

$$
\begin{array}{ll}
N_{2 i-1, m}^{*}=\frac{\delta_{2 i}+\alpha_{2 i+1} N_{2 i+1, m}^{*}}{\alpha_{2 i}} & (2 \leq i \leq k / 2-1) ; \\
N_{2 i, m}^{*} & =\frac{\alpha_{2 i-1} N_{2(i-1), m}^{*}-\delta_{2 i-1}}{\alpha_{2 i}}
\end{array}
$$

for odd $k$,

$$
\begin{aligned}
& N_{2 i, m}^{*}=\frac{\delta_{2 i+1}+\alpha_{2(i+1)} N_{2(i+1), m}^{*}}{\alpha_{2 i+1}} \quad(2 \leq i \leq(k-3) / 2) ; \\
& N_{2 i-1, m}^{*}=\frac{\alpha_{2(i-1)} N_{2 i-3, m}^{*}-\delta_{2(i-1)}}{\alpha_{2 i-1}} \quad(2 \leq i \leq(k+1) / 2) .
\end{aligned}
$$

As for the completely established state, we can get the corresponding relations by substituting $m$ for $k$ in the above. Making use of these relations (A1-A3), we can get the explicit formulas (3.1) and (3.2) of equilibrium values.

\section{B Proof of Theorem 1}

At first, we prove the following lemma:

Lemma 12. For any equilibrium state of the $m$ level system, if $N_{k, m}^{*}>0$ with some $k \leq m$, then $N_{i, m}^{*}>0$ for any $i<k$. 


\section{Proof of Lemma 12}

For even $k$, if $N_{k, m}^{*}>0$, then from (A2),

$$
N_{k, m}^{*}=\frac{\alpha_{k-1} N_{k-2, m}^{*}-\delta_{k-1}}{\alpha_{k}}>0 .
$$

This means that

$$
N_{k-2, m}^{*}>\frac{\delta_{k-1}}{\alpha_{k-1}}>0
$$

Then, again from (A2),

$$
\frac{\alpha_{k-3} N_{k-4, m}^{*}-\delta_{k-3}}{\alpha_{k-1}}>\frac{\delta_{k-1}}{\alpha_{k-1}}
$$

so that

$$
N_{k-4, m}^{*}>\frac{\alpha_{k-2} \delta_{k-1}}{\alpha_{k-3} \alpha_{k-1}}+\frac{\delta_{k-3}}{\alpha_{k-3}}>0 .
$$

By mathematical induction, we can prove that the equilibrium value of the $2 i$ th level is positive for any $i \leq k / 2$. Hence, from (A1) and (A2), as for the equilibrium value of the $2 i-1$ th level, we can easily find that it is positive for any $i$.

For odd $k$, from (A1) and (A3), we can easily find that the equilibrium value of the $2 i$ th level is positive. As for the $2 i-1$ th level, we can apply the analogous arguments as in case of even $k$. These arguments prove the lemma.

\section{Proof of Theorem 1}

When the $k$ level established state exists for an even $k$, making use of the similar arguments as those in the proof of Lemma 12, we can find that

$$
N_{2, m}^{*}>\frac{\delta_{3}}{\alpha_{3}}+\sum_{l=1}^{k / 2-2} \frac{\delta_{2 l+3}}{\alpha_{2 l+3}} \prod_{j=1}^{l} \frac{\alpha_{2 j+2}}{\alpha_{2 j+1}}
$$

for even $k$ if $N_{k, m}^{*}>0$. Then, from (A1), we have

$$
\frac{\phi}{\alpha_{2} N_{1, m}^{*}}-\frac{\delta_{1}}{\alpha_{2}}>\frac{\delta_{3}}{\alpha_{3}}+\sum_{l=1}^{k / 2-2} \frac{\delta_{2 l+3}}{\alpha_{2 l+3}} \prod_{j=1}^{l} \frac{\alpha_{2 j+2}}{\alpha_{2 j+1}} .
$$


From (3.1), this inequality leads to the following:

$$
\begin{aligned}
\phi & >\alpha_{2} Q_{k}\left(\frac{\delta_{3}}{\alpha_{3}}+\sum_{l=1}^{k / 2-2} \frac{\delta_{2 l+3}}{\alpha_{2 l+3}} \prod_{j=1}^{l} \frac{\alpha_{2 j+2}}{\alpha_{2 j+1}}+\frac{\delta_{1}}{\alpha_{2}}\right) \\
& =Q_{k}\left(\delta_{3} \frac{\alpha_{2}}{\alpha_{3}}+\sum_{l=1}^{k / 2-2} \delta_{2 l+3} \frac{\alpha_{2 l+2}}{\alpha_{2 l+3}} \prod_{j=1}^{l} \frac{\alpha_{2 j}}{\alpha_{2 j+1}}+\delta_{1}\right) \\
& =Q_{k}\left(\delta_{1}+\sum_{l=1}^{k / 2-1} \delta_{2 l+1} \prod_{j=1}^{l} \frac{\alpha_{2 j}}{\alpha_{2 j+1}}\right) .
\end{aligned}
$$

As $k$ is even, $k / 2-1=\llbracket(k-1) / 2 \rrbracket$. Consequently, from the definition of $P_{k}$, we can obtain the inequality that $\phi>P_{k} Q_{k}$. For odd $k$, we can carry out the similar arguments, and lastly prove the theorem.

\section{Proof of Corollaries 2 and 3}

\section{Proof of Corollary 2}

From the increasing monotonicity of $P_{k} Q_{k}$ in terms of $k$, we have $P_{j} Q_{j}<P_{k} Q_{k}$ for any $j<k$. From Theorem 1, if the $k$ level established state exists, we have $P_{k} Q_{k}<\phi$. Therefore, we have $P_{j} Q_{j}<\phi$ for any $j<k$. From Theorem 1, this means that any $j$ level established state with $j<k$ exists.

\section{Proof of Corollary 3}

For an even $k$, suppose that the $k-1$ level established state exists with $N_{k-1, m}^{*}>\delta_{k} / \alpha_{k}$. Then, from (A3),

$$
\frac{\alpha_{k-2} N_{k-3, m}^{*}-\delta_{k-2}}{\alpha_{k-1}}>\frac{\delta_{k}}{\alpha_{k}}
$$

that is,

$$
N_{k-3, m}^{*}>\frac{\delta_{k} \alpha_{k-1}}{\alpha_{k} \alpha_{k-2}}+\frac{\delta_{k-2}}{\alpha_{k-2}} .
$$

Hence, by mathematical induction, we can obtain the following:

$$
N_{1, m}^{*}>\frac{\delta_{2}}{\alpha_{2}}+\sum_{l=1}^{k / 2-1} \frac{\delta_{2(l+1)}}{\alpha_{2(l+1)}} \prod_{j=1}^{l} \frac{\alpha_{2 j+1}}{\alpha_{2 j}}=Q_{k} .
$$

As $k-1$ is odd, from (3.2) and the above inequality, we have $Q_{k}<\phi / P_{k-1}$. Now, we note that $\llbracket k / 2-1 \rrbracket=\llbracket(k-1) / 2 \rrbracket$ for even $k$. Thus, from the definition of $P_{k}$, we find that $P_{k-1}=P_{k}$ 
for even $k$. Consequently, we can find that $\phi>P_{k} Q_{k}$. Therefore, from Theorem 1 , the $k$ level established state exists for even $k$.

Inversely, for an even $k$, suppose that the $k$ level established state exists with $\phi>P_{k} Q_{k}$. Then, making use of the above arguments, we can derive the following inequality: $N_{1, m}^{*}>Q_{k}$. Then, from (A1) and the definition of $Q_{k}$, we can obtain the following:

$$
N_{3, m}^{*}>\frac{\delta_{4}}{\alpha_{4}}+\sum_{l=2}^{k / 2-1} \frac{\delta_{2(l+1)}}{\alpha_{2(l+1)}} \prod_{j=2}^{l} \frac{\alpha_{2 j+1}}{\alpha_{2 j}} .
$$

From (A2), by iterating the similar calculations, we can lastly find that $N_{k-1, m}^{*}>\delta_{k} / \alpha_{k}$. From Corollary 2, the $k-1$ level established state exists. Thus, we have proved Corollary 3 for any even $k$. We can carry out the similar arguments to prove Corollary 3 for any odd $k$.

\section{Proof of Theorem 4, Corollaries 5 and 6}

\section{Proof of Theorem 4}

For the $m$ level system, suppose that the $k(<m)$ level established state exists. Now, we define the following function $H_{k}$ :

$$
\begin{aligned}
& H_{k}\left(N_{1, m}, N_{2, m}, \ldots, N_{m, m}\right) \\
& =\sum_{i=1}^{k}\left[N_{i, m}-N_{i, m}^{*}\left(1+\log \frac{N_{i, m}}{N_{i, m}^{*}}\right)\right]+\sum_{i=k+1}^{m} N_{i, m},
\end{aligned}
$$

where $N_{i, m}^{*}$ for $i=1,2, \ldots, k$ is the positive equilibrium value at the $k$ level established state. It can be easily seen that $H_{k}$ is greater than zero for any $\left(N_{1, m}, \ldots, N_{m, m}\right)$ with positive $N_{i, m}$ for any $i$. $H_{k}$ equals to zero only for the $k$ level established state: $N_{i, m}=N_{i, m}^{*}$ for $i=1,2, \ldots, k$ and $N_{i, m}=0$ for $i=k+1, \ldots, m$.

Making use of (2.1) and (A1-A3),

$$
\frac{d H_{k}}{d t}=-\phi \frac{\left(N_{1, m}-N_{1, m}^{*}\right)^{2}}{N_{1, m} N_{1, m}^{*}}-\left(\delta_{k+1}-\alpha_{k+1} N_{k, m}^{*}\right) N_{k+1, m}-\sum_{i=k+2}^{m} \delta_{i} N_{i, m} .
$$

We can easily find that the first and the third terms of right side in (D2) are not positive for any $N_{i, m} \geq 0$. From the assumption of Theorem 4, the $k+1$ level established state does not exist. Thus, from Corollary 3, we have $N_{k, m}^{*} \leq \delta_{k+1} / \alpha_{k+1}$, that is, $\delta_{k+1}-\alpha_{k+1} N_{k, m}^{*} \geq 0$. This means that the second term of right side in (D2) is not positive, either. Therefore, we can obtain $d H_{k} / d t \leq 0$ for any $N_{i, m} \geq 0$. When $\left(N_{1, m}, \ldots, N_{m, m}\right)$ is at the state with $N_{1, m}=N_{1, m}^{*}$ and $N_{i, m}=0$ $(k+1 \leq i \leq m)$, then $d H_{k} / d t=0$. However, as long as $N_{i, m} \neq N_{i, m}^{*}$ for some $i$ such that $2 \leq i \leq k$, the dynamics (2.1) temporally changes the state so that $d H_{k} / d t$ eventually becomes negative unless the system reaches the $k$ level established state. These arguments show that $H_{k}$ is a Lyapunov function about the $k$ level established state, and eventually becomes zero as $t \rightarrow \infty$. Consequently, the $k$ level established state is globally stable. 


\section{Proof of Corollary 5}

As for the completely established state of the $m$ level system, we define the following function $H_{m}$ :

$$
H_{m}\left(N_{1, m}, N_{2, m}, \ldots, N_{m, m}\right)=\sum_{i=1}^{m}\left[N_{i, m}-N_{i, m}^{*}\left(1+\log \frac{N_{i, m}}{N_{i, m}^{*}}\right)\right],
$$

where $N_{i, m}^{*}(i=1,2, \ldots, m)$ is the positive equilibrium value at the completely established state. As in the proof of Theorem 4, we can prove that $H_{m}$ is non-negative for any $\left(N_{1}, \ldots, N_{m}\right)$ with positive value of $N_{i, m}$ for any $i$, and equals to zero only at the completely established state, and eventually becomes zero as $t \rightarrow 0$. This argument means that $H_{m}$ given by (D3) is a Lyapunov function about the completely established state. These arguments prove Corollary 5 .

\section{Proof of Corollary 6}

We can obtain the characteristic equation for the $k$ level established state of the $m$ level system:

$$
\left(-\delta_{m}-\lambda\right)\left(-\delta_{m-1}-\lambda\right) \cdots\left(-\delta_{k+2}-\lambda\right)\left(\alpha_{k+1} N_{k, m}^{*}-\delta_{k+1}-\lambda\right) J_{k}=0
$$

with

$$
J_{k}=\left|\begin{array}{ccccc}
A_{1}-\lambda & -C_{1} & 0 & \ldots & 0 \\
B_{2} & A_{2}-\lambda & \ddots & \ddots & \vdots \\
0 & \ddots & \ddots & -C_{k-2} & 0 \\
\vdots & \ddots & B_{k-1} & A_{k-1}-\lambda & -C_{k-1} \\
0 & \ldots & 0 & B_{k} & A_{k}-\lambda
\end{array}\right|,
$$

where

$$
\begin{aligned}
& A_{1}=-\delta_{1}-\alpha_{2} N_{2, m}^{*} ; \\
& A_{i}=0 \quad(i=2,3, \ldots, k) ; \\
& B_{i}=\alpha_{i} N_{i, m}^{*} \quad(i=1,2, \ldots, k) ; \\
& C_{i}=\alpha_{i+1} N_{i, m}^{*} \quad(i=1,2, \ldots, k) .
\end{aligned}
$$

Eigenvalues $-\delta_{k+2},-\delta_{k+3}, \ldots$, and $-\delta_{m}$ are all negative. If the eigenvalue $\alpha_{k+1} N_{k, m}^{*}-\delta_{k+1} \leq 0$, that is, if $N_{k, m}^{*} \leq \delta_{k+1} / \alpha_{k+1}$, from Corollary 3, the $k+1$ level established state does not exist. Hence, from Theorem 4 , the $k$ level established state becomes globally stable. This is contradictory for the assumption. Therefore, $N_{k, m}^{*}>\delta_{k+1} / \alpha_{k+1}$, that is, the eigenvalue $\alpha_{k+1} N_{k, m}^{*}-\delta_{k+1}$ is positive. From Corollary 3, we lastly prove Corollary 6.

\section{E Proof of Lemma 8}

We can obtain the following characteristic equation for the $m$ level established state of the $m+1$ level system:

$$
\left(\alpha_{m+1} N_{m, m+1}^{*}-\delta_{m+1}-\lambda\right) J_{m}=0
$$


where $J_{m}$ is defined similarly to $J_{k}$ in Appendix D, substituting $m$ for $k$ with

$$
\begin{aligned}
& A_{i}=\alpha_{i} N_{i-1, m+1}^{*}-\delta_{i}-\alpha_{i+1} N_{i+1, m+1}^{*} \quad(i=2,3, \ldots, m) \\
& A_{m+1}=\alpha_{m+1} N_{m, m+1}^{*}-\delta_{m+1} .
\end{aligned}
$$

$B_{i}$ and $C_{i}(i=1,2, \ldots, k)$ are defined the same as in Appendix D.

When the completely established state of the $m$ level system exists, suppose that the $m+1$ th trophic level with

$$
\frac{\delta_{m+1}}{\alpha_{m+1}}<N_{m, m}^{*}
$$

is introduced into the system. For the $m$ level established state of the $m+1$ level system, the value $N_{m, m+1}^{*}$ equals to the value $N_{m, m}^{*}$ at the completely established state of the $m$ level system. Hence, from (E2), $\delta_{m+1} / \alpha_{m+1}<N_{m, m+1}^{*}$. Therefore, $\alpha_{m+1} N_{m, m+1}^{*}-\delta_{m+1}>0$. From (E1), this means that the eigenvalue $\alpha_{m+1} N_{m, m+1}^{*}-\delta_{m+1}$ is positive. Lastly, the $m$ level established state is unstable.

\section{F Number of trophic levels in the longest chain}

In case of $q<1$ and $\phi<P Q$ for even $m$, if

$$
\phi>P_{m} Q_{m}=\frac{\delta_{1} \delta_{2}}{\alpha_{2}}\left(\frac{1-q^{m / 2}}{1-q}\right)^{2},
$$

then, from Theorem 1, the $m$ level established state exists. From this inequality, we have $m<\Theta$, where $\Theta$ is given by (5.5). From $\phi<P Q=\delta_{1} \delta_{2} / \alpha_{2} /(1-q)^{2}$, we have $(\rho-2) / \rho<q$ with $\rho=\sqrt{4 \alpha_{2} \phi /\left(\delta_{1} \delta_{2}\right)}$.

For odd $m$, if

$$
\phi>P_{m} Q_{m}=\frac{\delta_{1} \delta_{2}}{\alpha_{2}(1-q)^{2}}\left(1-q^{(m+1) / 2}\right)\left(1-q^{(m-1) / 2}\right)
$$

then the $m$ level established state exists. From this inequality, we have $m<\Lambda$ where $\Lambda$ is given by (5.6).

Now, we define $m_{\text {even }}^{*}$ as the maximal even number which satisfies that $m_{\text {even }}^{*}<\Theta$ and $m_{\text {odd }}^{*}$ as the maximal odd number which satisfies that $m_{\text {odd }}^{*}<\Lambda$. We can obtain the upper limit $m_{\max }$ of the number of trophic levels by $m_{\max }=\max \left\{m_{\text {even }}^{*}, m_{\text {odd }}^{*}\right\}$.

First we prove that $0 \leq \Lambda-\Theta<1$. From (5.5) and (5.6), we can find that $\Lambda-\Theta \geq 0$ is equivalent to $(\rho q-\rho+2)^{2} \geq 0$. On the other hand, we can find that $\Lambda-\Theta<1$ is equivalent to $\phi<P Q$ when $q<1$. Therefore, we finally obtain $0 \leq \Lambda-\Theta<1$. This means $\llbracket \Lambda \rrbracket-\llbracket \Theta \rrbracket=0$ or 1 .

For even $\llbracket \Theta \rrbracket$, if $\llbracket \Lambda \rrbracket=\llbracket \Theta \rrbracket$, then $m_{\text {even }}^{*}=\llbracket \Theta \rrbracket=\llbracket \Lambda \rrbracket$ and $m_{\text {odd }}^{*}=\llbracket \Lambda \rrbracket-1$. Hence, $m_{\max }=$ $m_{\text {even }}^{*}=\llbracket \Lambda \rrbracket$. If $\llbracket \Lambda \rrbracket=\llbracket \Theta \rrbracket+1$, then $m_{\text {even }}^{*}=\llbracket \Theta \rrbracket=\llbracket \Lambda \rrbracket-1$ and $m_{\text {odd }}^{*}=\llbracket \Lambda \rrbracket$. Hence, $m_{\max }=m_{\text {odd }}^{*}=\llbracket \Lambda \rrbracket$. Lastly, for even $\llbracket \Theta \rrbracket$, we can obtain $m_{\max }=\llbracket \Lambda \rrbracket$. For odd $\llbracket \Theta \rrbracket$, if $\llbracket \Lambda \rrbracket=\llbracket \Theta \rrbracket$, 
then $m_{\text {even }}^{*}=\llbracket \Theta \rrbracket-1$ and $m_{\text {odd }}^{*}=\llbracket \Lambda \rrbracket=\llbracket \Theta \rrbracket$. Hence, $m_{\max }=m_{\text {odd }}^{*}=\llbracket \Theta \rrbracket$. If $\llbracket \Lambda \rrbracket=\llbracket \Theta \rrbracket+1$, then $m_{\text {even }}^{*}=\llbracket \Theta \rrbracket-1$ and $m_{\text {odd }}^{*}=\llbracket \Lambda \rrbracket-1=\llbracket \Theta \rrbracket$. Hence, $m_{\max }=m_{\text {odd }}^{*}=\llbracket \Theta \rrbracket$. Lastly, for odd $\llbracket \Theta \rrbracket$, we can obtain $m_{\max }=\llbracket \Theta \rrbracket$. Similar arguments are applicable for the case when $q>1$.

In case of $q=1$ for even $m$, from Theorem 1 , if the following inequality is satisfied, then the $m$ level established state exists:

$$
\begin{aligned}
\phi> & \left(\delta_{1}+\sum_{l=1}^{m / 2-1} \delta_{1} \xi^{2 l} \prod_{j=1}^{l} \frac{1}{\zeta}\right)\left(\frac{\delta_{2}}{\alpha_{2}}+\sum_{l=1}^{m / 2-1} \frac{\delta_{2} \xi^{2 l}}{\alpha_{2} \zeta^{2 l}} \prod_{j=1}^{l} \zeta\right) \\
= & \frac{\delta_{1} \delta_{2}}{\alpha_{2}}\left(1+\sum_{l=1}^{m / 2-1} q^{l}\right)^{2} \\
= & \left(\frac{m}{2}\right)^{2} \frac{\delta_{1} \delta_{2}}{\alpha_{2}}
\end{aligned}
$$

that is, $m<\rho$. For odd $m$, if

$$
\phi>\frac{\delta_{1} \delta_{2}}{\alpha_{2}}\left(1+\sum_{l=1}^{(m-1) / 2} q^{l}\right)\left(1+\sum_{l=1}^{(m-3) / 2} q^{l}\right)=\left(m^{2}-1\right) \frac{\delta_{1} \delta_{2}}{4 \alpha_{2}},
$$

then the $m$ level established state exists. This condition is equivalent to $m<\sqrt{\rho^{2}+1}$. Lastly, we can obtain (5.7) with the similar arguments as in case of $q<1$.

\section{G Proof of Theorem 10}

Before the proof of Theorem 10, we prove the following lemma:

Lemma 13. If $\alpha_{i}=\alpha$ and $\delta_{i}=\delta(i=1,2, \ldots, m)$, it is always satisfied that $N_{i-1, m}^{*}>N_{i, m}^{*}$ for any $i \leq m$ at the completely established state of the $m$ level system.

\section{Proof of Lemma 13}

In case of even $m$, from (3.1) and (A1), we can derive

$$
N_{2 i+1, m}^{*}=\left(\frac{m}{2}-i\right) \frac{\delta}{\alpha} \quad(0 \leq i \leq m / 2-1),
$$

and similarly from (A2),

$$
N_{2(i-1), m}^{*}=N_{2 i, m}^{*}+\frac{\delta}{\alpha} \quad(0 \leq i \leq m / 2)
$$


From these relationships, we obtain

$$
N_{2 i, m}^{*}=\left(\frac{m}{2}-i\right) \frac{\delta}{\alpha}+N_{m, m}^{*} \quad(0 \leq i \leq m / 2) .
$$

In addition, from (3.1) and the definition of $P_{k}$ and $Q_{k}$, we have

$$
N_{m-1, m}^{*}-N_{m, m}^{*}=\frac{\delta}{\alpha}-N_{m, m}^{*}>0
$$

So, from (G1-G3), we have

$$
\begin{aligned}
& N_{2 i, m}^{*}-N_{2 i+1, m}^{*}=N_{m, m}^{*}>0 \quad(1 \leq i \leq m / 2-1) ; \\
& N_{2 i-1, m}^{*}-N_{2 i, m}^{*}=\frac{\delta}{\alpha}-N_{m, m}^{*}>0 \quad(1 \leq i \leq m / 2) .
\end{aligned}
$$

Consequently, we obtain that $N_{i-1, m}^{*}>N_{i, m}^{*}$ for any $i \leq m$ at the completely established state of the $m$ level system. We can obtain the same result in case of odd $m$, applying the similar arguments. Finally, these arguments prove the lemma.

\section{Proof of Theorem 10}

From the assumption of Theorem 10, for a sufficiently narrow range of $\alpha_{i}$ and $\delta_{i}$, we can define a sufficiently small positive value $\varepsilon=\max \left\{\varepsilon_{\alpha}, \varepsilon_{\delta}\right\}$, where

$$
\sup _{i, j}\left|\alpha_{i}-\alpha_{j}\right|=\varepsilon_{\alpha} ; \quad \sup _{i, j}\left|\delta_{i}-\delta_{j}\right|=\varepsilon_{\delta}
$$

Then let us denote $\alpha_{i}=\bar{\alpha}+O(\varepsilon)$ and $\delta_{i}=\bar{\delta}+O(\varepsilon)$, where $\bar{\alpha}$ and $\bar{\delta}$ are the mean values of $\alpha_{i}$ and $\delta_{i}$ respectively over all trophic levels in the $m_{\max }$ level established state.

For even $m_{\max }$, in the same way as in the proof of Lemma 13, we can derive the followings from (3.1), (A1) and (A2):

$$
\begin{aligned}
& N_{2 i+1, m_{\max }}^{*}=\left(\frac{m_{\max }}{2}-i\right) \frac{\bar{\delta}}{\bar{\alpha}}+O(\varepsilon) \quad\left(0 \leq i \leq m_{\max } / 2-1\right) ; \\
& N_{2 i, m_{\max }}^{*}=\left(\frac{m_{\max }}{2}-i\right) \frac{\bar{\delta}}{\bar{\alpha}}+\bar{N}_{m_{\max }, m_{\max }}^{*}+O(\varepsilon) \\
& \quad\left(0 \leq i \leq m_{\max } / 2\right),
\end{aligned}
$$

where $\bar{N}_{i, m_{\max }}^{*}\left(i=1,2, \ldots, m_{\max }\right)$ is the equilibrium value at the $m_{\max }$ level established state with $\alpha_{i}=\bar{\alpha}$ and $\delta_{i}=\bar{\delta}$ for any $i$. Then, from (G4) and (G5), we have

$$
\begin{aligned}
& N_{2 i, m_{\max }}^{*}-N_{2 i+1, m_{\max }}^{*}= \bar{N}_{m_{\max }, m_{\max }}^{*}+O(\varepsilon)>0 \\
& N_{2 i-1, m_{\max }}^{*}-N_{2 i, m_{\max }}^{*}=\frac{\bar{\delta}}{\bar{\alpha}}-\bar{N}_{m_{\max }, m_{\max }}^{*}+O(\varepsilon) \\
& \quad\left(1 \leq i \leq m_{\max } / 2\right) .
\end{aligned}
$$


From Lemma 13, we find that

$$
\bar{N}_{m_{\max }-1, m_{\max }}^{*}=\frac{\bar{\delta}}{\bar{\alpha}}>\bar{N}_{m_{\max }, m_{\max }}^{*} .
$$

Thus, from (G7), for sufficiently small $\varepsilon$, we have

$$
N_{2 i-1, m_{\max }}^{*}-N_{2 i, m_{\max }}^{*}>0 \quad\left(1 \leq i \leq m_{\max } / 2\right) .
$$

From (G6) and (G8), we finally obtain $N_{i-1, m_{\max }}^{*}>N_{i, m_{\max }}^{*}$ for any $i \leq m_{\max }$ at the $m_{\max }$ level established state in case of sufficiently narrow ranges of $\alpha_{i}$ and $\delta_{i}$. We can carry out the same arguments for odd $m_{\max }$. These arguments prove Theorem 10.

\section{H Proof of Corollary 11}

For the proof of Corollary 11, we prove the following lemma at first:

Lemma 14. At the completely established state of the $m$ level system with $\alpha_{i}=\alpha$ and $\delta_{i}=\delta$ for any $i$, after the $k$ th level $(4 \leq k \leq m)$ is removed, the system transits to the $k-1$ level established state with an energy reserve distribution which satisfies the following characteristics: For even $k$,

$$
\begin{aligned}
& N_{2(i-1), k-1}^{*}<N_{2 i-1, k-1}^{*} \quad(2 \leq i \leq k / 2) ; \\
& N_{2(i-1), k-1}^{*}>N_{2 i, k-1}^{*} \quad(2 \leq i \leq k / 2-1) \text {; } \\
& N_{2 i-1, k-1}^{*}>N_{2 i+1, k-1}^{*} \quad(1 \leq i \leq k / 2-1),
\end{aligned}
$$

and for odd $k$,

$$
\begin{array}{cccl}
N_{2 i-1, k-1}^{*} & < & N_{2 i, k-1}^{*} & (1 \leq i \leq(k-1) / 2) \\
N_{2(i-1), k-1}^{*} & > & N_{2 i, k-1}^{*} & (2 \leq i \leq(k-1) / 2) \\
N_{2 i-1, k-1}^{*} & > & N_{2 i+1, k-1}^{*} & (1 \leq i \leq(k-3) / 2)
\end{array}
$$

\section{Proof of Lemma 14}

Since the completely established state of $m_{\text {max }}$ level system exists, $P_{m} Q_{m}<\phi$. Now, suppose that the value of $N_{k, m}$ for a $k$ th trophic level $(3 \leq k \leq m)$ is changed to zero. This corresponds to the removal of the $k$ th trophic level. From Lemma 12 in Appendix B, Corollaries 2 and 5, the system transits to the $k-1$ level established state.

At the $k-1$ level established state for even $k$, from (A1) and (A3), we have

$$
\begin{aligned}
N_{2 i-3, k-1}^{*} & =N_{2 i-1, k-1}^{*}+\frac{\delta}{\alpha} \quad(2 \leq i \leq k / 2) ; \\
N_{2 i, k-1}^{*} & =N_{2(i+1), k-1}^{*}+\frac{\delta}{\alpha} \quad(1 \leq i \leq k / 2-2),
\end{aligned}
$$


where $N_{i, k-1}^{*}$ is the equilibrium value of the $i$ th trophic level at the $k-1$ level established state with $\alpha_{i}=\alpha$ and $\delta_{i}=\delta$. Hence, in terms of the even (resp. odd) levels, the equilibrium value of each level is greater than that of any higher level.

From (H1), we have

$$
N_{2 i-1, k-1}^{*}=\left(\frac{k}{2}-i\right) \frac{\delta}{\alpha}+N_{k-1, k-1}^{*} \quad(1 \leq i \leq k / 2) .
$$

On the other hand, from (A1) and (H2),

$$
N_{2 i, k-1}^{*}=\left(\frac{k}{2}-i\right) \frac{\delta}{\alpha} \quad(1 \leq i \leq k / 2-1) .
$$

Thus, from (H3) and (H4),

$$
\begin{aligned}
N_{2 i-1, k-1}^{*}-N_{2(i-1), k-1}^{*} & =N_{k-1, k-1}^{*}-\frac{\delta}{\alpha}>0 \quad(2 \leq i \leq k / 2) ; \\
N_{2 i-1, k-1}^{*}-N_{2 i, k-1}^{*} & =N_{k-1, k-1}^{*}>0 \quad(1 \leq i \leq k / 2-1),
\end{aligned}
$$

where the right side of (H5) is positive from Corollary 3.

For odd $k$, from (A1) and (A2), we can obtain the similar results that the equilibrium value of each level is greater than that of any higher level in terms of the even (resp. odd) levels, and the following relations for $k \geq 5$ :

$$
\begin{aligned}
& N_{2 i, k-1}^{*}-N_{2 i-1, k-1}^{*}=N_{k-1, k-1}^{*}-\frac{\delta}{\alpha}>0 \quad(1 \leq i \leq(k-1) / 2) ; \\
& N_{2 i, k-1}^{*}-N_{2 i+1, k-1}^{*}=N_{k-1, k-1}^{*}>0 \quad(1 \leq i \leq(k-3) / 2) .
\end{aligned}
$$

When $k=3$, we have $N_{1,2}^{*}=\delta / \alpha$ and $N_{2,2}^{*}=\phi / \delta-\delta / \alpha$ from (A1). Then, we can derive

$$
N_{2,2}^{*}-N_{1,2}^{*}=\frac{\phi}{\text { delta }}-\frac{2 \delta}{\alpha}>0
$$

from the existence of the three level established state, that is, $\phi>\bar{P}_{3} \bar{Q}_{3}=2 \delta^{2} / \alpha$. These arguments prove the lemma.

\section{Proof of Corollary 11}

Now, we consider the case of $\alpha_{i}=\bar{\alpha}+O(\varepsilon)$ and $\delta_{i}=\bar{\delta}+O(\varepsilon)$, where $\bar{\alpha}$ and $\bar{\delta}$ are the mean values of $\alpha_{i}$ and $\delta_{i}$ respectively. The definition of $\varepsilon$ is same as in the proof of Theorem 10 (Appendix G). Applying the similar arguments as in the proof of Lemma 14, for even $k$, we can derive

$$
\begin{aligned}
N_{2 i-1, k-1}^{*} & =\left(\frac{k}{2}-i\right) \frac{\bar{\delta}}{\bar{\alpha}}+\bar{N}_{k-1, k-1}^{*}+O(\varepsilon) \quad(1 \leq i \leq k / 2) ; \\
N_{2 i, k-1}^{*} & =\left(\frac{k}{2}-i\right) \frac{\bar{\delta}}{\bar{\alpha}}+O(\varepsilon) \quad(1 \leq i \leq k / 2-1) .
\end{aligned}
$$


Then, we have

$$
\begin{aligned}
N_{2 i-1, k-1}^{*}-N_{2(i-1), k-1}^{*} & =\bar{N}_{k-1, k-1}^{*}-\frac{\bar{\delta}}{\bar{\alpha}}+O(\varepsilon) \quad(2 \leq i \leq k / 2) ; \\
N_{2 i-1, k-1}^{*}-N_{2 i, k-1}^{*} & =\bar{N}_{k-1, k-1}^{*}+O(\varepsilon) \quad(1 \leq i \leq k / 2-1) .
\end{aligned}
$$

From Corollary 3, we have $\bar{N}_{k-1, k-1}^{*}>\bar{\delta} / \bar{\alpha}$. Therefore, for a sufficiently narrow range of $\alpha_{i}$ and $\delta_{i}$, the right hands of (H9) and (H10) are positive. These arguments can be applied for odd $k$, either. These arguments prove Corollary 11.

\section{Pyramid shape in case of a geometric variation of $\alpha_{i}$ and $\delta_{i}$}

Let us consider the possibly longest chain with $P_{m_{\max }+1} Q_{m_{\max }+1}>\phi>P_{m_{\max }} Q_{m_{\max }}$. For even $m_{\max }$ with $q \neq 1$, from (3.1), we have

$$
\begin{aligned}
& N_{2 i, m_{\max }}^{*}-N_{2 i+1, m_{\max }}^{*} \\
& \quad=\frac{1}{\alpha_{2} \zeta^{i-1} Q_{m_{\max }}}\left\{\phi-\frac{1-q^{i}+\left(q^{i}-q^{m_{\max } / 2}\right) \xi / \zeta}{1-q} \delta_{1} Q_{m_{\max }}\right\} .
\end{aligned}
$$

Assuming $\xi / \zeta \leq 1$, we can find

$$
P_{m_{\max }}-\frac{1-q^{i}+\left(q^{i}-q^{m_{\max } / 2}\right) \xi / \zeta}{1-q} \delta_{1}=\left(1-\frac{\xi}{\zeta}\right) q^{i} P_{m_{\max }-2 i} \geq 0 .
$$

Thus, from (I1),

$$
N_{2 i, m_{\max }}^{*}-N_{2 i+1, m_{\max }}^{*} \geq \frac{1}{\alpha_{2} \zeta^{i-1} Q_{m_{\max }}}\left(\phi-P_{m_{\max }} Q_{m_{\max }}\right)>0 .
$$

Next, from (3.1), we have

$$
\begin{aligned}
& N_{2 i-1, m_{\max }}^{*}-N_{2 i, m_{\max }}^{*}= \\
& \frac{1}{\alpha_{2} \zeta^{i-1} Q_{m_{\max }}}\left\{\frac{1-q^{i}+\left(q^{i-1}-q^{m_{\max } / 2}\right) \xi}{1-q} \delta_{1} Q_{m_{\max }+1}-\phi\right\},
\end{aligned}
$$

where we used the relation that $Q_{m_{\max }}=Q_{m_{\max }+1}$ for even $m_{\max }$. Then, we have

$$
\frac{1-q^{i}+\left(q^{i-1}-q^{m_{\max } / 2}\right) \xi}{1-q} \delta_{1}-P_{m_{\max }+1}=\xi q^{i-1}\left(1-\frac{\xi}{\zeta}\right) P_{m_{\max }-2(i-1)} \geq 0 .
$$

Hence, from (I2), we obtain

$$
N_{2 i-1, m_{\max }}^{*}-N_{2 i, m_{\max }}^{*} \geq \frac{1}{\alpha_{2} \zeta^{i-1} Q_{m_{\max }}}\left\{P_{m_{\max }+1} Q_{m_{\max }+1}-\phi\right\}>0 .
$$

Therefore, we prove that $N_{i-1, m_{\max }}^{*}>N_{i, m_{\max }}^{*}$ for $2 \leq i \leq m_{\max } / 2$. We can carry out the same arguments for odd $m_{\max }$. Also for any $m_{\max }$ with $q=1$, we can apply the similar arguments. 


\section{$\mathbf{J}$ Sufficiently long chain in case of a geometric variation of $\alpha_{i}$ and $\delta_{i}$}

From (3.1), for even $m \gg 1$ when $q<1$ and $\phi>P Q$, we have the following energy reserves at the $m$ level established state:

$$
\begin{aligned}
N_{2 i, m}^{*} & \approx \frac{1}{\zeta^{i-1}}\left[\frac{\phi(1-q)}{\delta_{1} \xi}-\frac{\delta_{1}\left(1-q^{i}\right)}{\alpha_{2}(1-q)}\right] \quad(2 \leq i \leq m / 2) \\
N_{2 i+1, m}^{*} & \approx \frac{\delta_{2}}{\alpha_{2}(1-q)}\left(\frac{\xi}{\zeta}\right)^{2 i} \quad(1 \leq i \leq m / 2-1) \\
N_{2, m}^{*} & \approx \frac{\phi(1-q)}{\delta_{1} \xi}-\frac{\delta_{1}}{\alpha_{2}} \\
N_{1, m}^{*} & \approx \frac{\delta_{1} \xi}{\alpha_{2}(1-q)} .
\end{aligned}
$$

Thus, we can obtain the following approximate ratio between $N_{2 i, m}^{*}$ and $N_{2 i+1, m}^{*}$ :

$$
\frac{N_{2 i+1, m}^{*}}{N_{2 i, m}^{*}} \approx \frac{\delta_{1}^{2} q^{i+1}}{\phi \alpha_{2}(1-q)^{2}-\delta_{1}^{2} \xi\left(1-q^{i}\right)}=\frac{P Q q^{i} \xi / \zeta}{\phi-P Q\left(1-q^{i}\right)} .
$$

From $q<1$ and $\phi>P Q$, we can find that this value is positive for any $i$. For sufficiently large $i$, this ratio is smaller than 1 . In contrast, we have

$$
\frac{N_{2 i, m}^{*}}{N_{2 i-1, m}^{*}} \approx \frac{\phi \alpha_{2}(1-q)^{2}-\delta_{1}^{2} \xi\left(1-q^{i}\right)}{\delta_{1}^{2} \zeta q^{i}}=\frac{\phi-P Q\left(1-q^{i}\right)}{P Q q^{i} \zeta / \xi} .
$$

This value is also positive and larger than 1 for sufficiently large $i$. We can apply the same arguments for odd $m$. Therefore, any pyramid shape of the energy reserve distribution could not appear in any sufficiently long chain when $q<1$ and $\phi>P Q$.

\section{REFERENCES}

[1] E.B. Beisner, E. McCauley, F.J. Wrona. The influence of temperature and food chain length on plankton predator-prey dynamics. Can. J. Fish. Aquat. Sci., 54 (1997), 586-595.

[2] J.E. Cohen, T. Jonsson, S.R. Carpenter. Ecological community description using the food web, species abundance, and body size. Proc. Natl. Acad. Sci. USA, 100 (2003), 1781-1786.

[3] D.L. DeAngelis, W.M. Post, G. Sugihara (eds.). Current Trends in Food Web Theory Report on a Food Web Workshop. ORNL-5983, Oak Ridge National Laboratory, Oak Ridge, 1983. 
[4] B. Drossel, A. McKane. Modelling food web. in: S. Bornholdt, H.G. Schuster (eds.). Handbook of Graphs and Networks. Wiley-VCH, Berlin, 2003.

[5] D. Gascuel.The trophic level based model: A theoretical approach of fishing effects on marine ecosystems. Ecol. Mod., 189 (2005), 315-332.

[6] T. Gross, W. Ebenhöh, U. Feudel. Long food chains are in general chaotic. Oikos, 109 (2005), $135-144$.

[7] W.S.C. Gurney, R.M. Nisbet.Ecological Dynamics. Oxford University Press, Oxford, 1998.

[8] S.J. Hall, D.G. Raffaelli. Food webs - theory and reality. Adv. Ecol. Res., 24 (1993), 187239.

[9] G.W. Harrison. Global stability of food chains. Am. Nat., 114 (1979), 455-457.

[10] M. Higashi, T.P. Burns, B.C. Patten. Food network unfolding: an extension of trophic dynamics for application to natural ecosystems. J. theor. Biol., 140 (1989), 243-261.

[11] G.E. Hutchinson.An Introduction to Population Ecology. Yale University, New Haven, CT, 1978.

[12] B. Jenkins, R.L. Kitching, S.L. Pimm. Productivity, disturbance, and food web structure at a local spatial scale in experimental container habitats. Oikos, 65 (1992), 249-255.

[13] F. Jordán, I. Scheuring, I. Molnár. Persistence and flow reliability in simple food webs. Ecol. Mod., 161 (2003), 117-124.

[14] B.W. Kooi, M.P. Boer, S.A.L.M. Kooijman. Resistance of a food chain to invasion by a top predator. Math. Biosci., 157 (1999), 217-236.

[15] R.L. Lindeman. The trophic-dynamic aspect of ecology. Ecology, 23 (1942), 399-418.

[16] E.P. Odum. Fundamentals of Ecology, 3rd ed. W.B. Saunders Co., Philadelphia, 1971.

[17] L. Oksanen, T. Oksanen, P. Ekerholm, J. Moen, P.A. Lundberg, M. Schneider, M. Aunapuu. Structure and dynamics of arctic-subarctic grazing webs in relation to primary production. in: G.A. Polis, K.O. Winemiller, K.O. (eds.). Food Webs: Integration of Pattern and Process. Chapman and Hall, New York, 1996.

[18] S.L. Pimm. Food Webs. Chapman and Hall, New York, 1982.

[19] S.L. Pimm, J.H. Lawton. Number of trophic levels in ecological communities. Nature, 268 (1977), 329-331.

[20] S.L. Pimm, R.L. Kitching. The determinants of food chain length. Oikos, 50 (1987), 302-307. 
[21] S.L. Pimm, J.H. Lawton, J.E. Cohen. Food web patterns and their consequences. Nature, 350 (1991), 669-674.

[22] G.A. Polis. Complex Trophic Interactions in Deserts: An empirical crituque of food-web theory. Am. Nat., 138 (1991), 123-155.

[23] D.M. Post. The long and short of food-chain length. Trends Ecol. Evol., 17 (2002), 269-277.

[24] D.M. Post. Using stable isotopes to estimate trophic position: Models, methods, and assumptions. Ecology, 83 (2002), 703-718.

[25] D.M. Post, M.L. Pace, N.G.Jr Hairston. Ecosystem size determines food-chain length in lakes. Nature, 405 (2000), 1047-1049.

[26] T.W. Schoener. Food webs from the small to the large. Ecology, 70 (1989), 1559-1589.

[27] M. Spencer, P.H. Warren. The effect of habitat size and productivity on food web structure in small aquatic microcosms. Oikos, 75 (1996), 419-430.

[28] G. Sugihara. Holes in niche space: a derived assembly rule and its relation to intervality. in: D.L. DeAngelis, W.N. Post, G. Sugihara (eds.). Current Trends in Food Web Theory. Oak Ridge National Laboratory, Oak Ridge, 1983.

[29] G. Sugihara. Graph theory, homology and food webs. Proc. Symp. App. Math., 30 (1984), 83-101.

[30] G. Sugihara, L.-F. Bersier, K. Schoenly. Effects of taxonomic and trophic aggregation on food web properties. Oecologia, 112 (1997), 272-284.

[31] Yu.M. Svirezhev, D.O. Logofet. Stability of Biological Communities. Mir Publishers, Moscow, 1983.

[32] E. Teramoto. Dynamical structure of food chains and energy trophic levels. Jpn. J. Ecol., 43 (1993), 21-29. (Japanese with English abstract)

[33] R.E. Ulanowicz. Identifying the structure of cycling in ecosystems. Math. Biosci., 65 (1983), 219-237.

[34] R.J. Williams, N.D. Martinez. Simple rules yield complex food webs. Nature, 404 (2000), 180-183.

[35] P. Yodzis. Energy flow and the vertical structure of real ecosystems. Oecologia, 65 (1984), $86-88$.

[36] M.J.V. Zanden, B.J. Shuter, N. Lester, J.B. Rasmussen. Patterns of food chain length in lakes: A stable isotope study. Am. Nat., 154 (1999), 406-416. 